\title{
WIK Zebrafish
}

National Cancer Institute

\section{Source}

National Cancer Institute. WIK Zebrafish. NCI Thesaurus. Code C79954.

A wild-type zebrafish line that is polymorphic relative to the Tubingen line. 